\title{
Effect on hospital length of stay of tourniquet use during internal fixation of ankle fracture: randomized controlled trial
}

Julius Sim MSc PhD, ${ }^{1}$ Natalie Grocott BSc, ${ }^{2}$ Haroon Majeed MSc FRCS, ${ }^{2}$ Damian McClelland MB ChB FRCS ${ }^{2}$

1. Institute for Primary Care and Health Sciences, Keele University, Staffordshire ST5 $5 B G, U K$

2. University Hospitals of North Midlands, Royal Stoke University Hospital, Staffordshire ST4 6QG, UK

\begin{abstract}
Approximately $50 \%$ of ankle fractures require internal fixation. A tourniquet is often employed to create a bloodless operative field, but its effect on hospital length of stay (LOS) is unclear. This paper reports a parallel-group observer-blinded randomized trial of the effect of tourniquet use on LOS. 188 adult patients requiring internal fixation of ankle fracture were randomized to surgery with or without tourniquet. LOS was analyzed on intention-to-treat principles using Poisson regression. As a secondary outcome, duration of operation was analyzed by ANCOVA. Mean LOS was $1.79 \pm 1.50$ days in the tourniquet group and $1.65 \pm 1.11$ days in the no-tourniquet group. The covariate-adjusted rate ratio for LOS (reference group: no tourniquet) was 1.084 (95\% CI 0.871, 1.348; $p=$ .470 ). Sensitivity analyses (unadjusted intention-to-treat analysis and as-treated analysis) gave similar non-significant results. The covariate-adjusted mean difference in duration of operation was 3.03 minutes (95\% CI -4.96, 11.02; $p=.455)$, favoring the tourniquet group; sensitivity analyses again gave similar results. Adverse events did not differ significantly between groups. In conclusion, the use of a tourniquet during internal fixation does not significantly influence hospital LOS, of which pre-injury medical condition of the patient appears to be the most important determinant.
\end{abstract}

\section{Level of clinical evidence: 1}

Keywords: ankle fracture, operative fixation, tourniquet, length of stay, randomized controlled trial 


\section{Introduction}

Ankle fractures are common injuries, accounting for approximately $9 \%$ of all fractures and occuring at a rate of $187 / 100,000$ people each year [1]. This equates to roughly 121,000 ankle fractures in the UK each year. Approximately half are deemed to be unstable and subsequently require operative fixation. Operative management is commonly carried out using a tourniquet to create a bloodless field [2]; however, tourniquet use during lower-limb surgery has been associated with a range of adverse events, including vascular injury, post-operative swelling, joint stiffness, increased postoperative pain, neurapraxia, increased risk of infection and deep vein thrombosis $[3,4]$. In a recent systematic review of four studies, tourniquet use in foot and ankle surgery was also shown to lead to an increased length of hospital stay [5].

If the use of a tourniquet delays recovery following fixation of ankle fracture, this will be evidenced by a longer hospital stay, as previously reported [6,7]. Accordingly, the objective of this multi-centre, parallel-group randomised study was to determine whether the use of a tourniquet during surgery for patients with closed ankle fractures affected the hospital LOS.

\section{Patients and methods}

\section{Recruitment and Randomization}

Adult patients with closed ankle fractures were invited to take part in a randomized trial of tourniquet versus no tourniquet use for the operative management of ankle fractures. Patients were recruited from the University Hospital of North Midlands (UHNM) NHS trust, Cambridge University Hospitals NHS Trust, and the Princess Royal Hospital, Telford, between 2012 and 2016. In total 471 patients were assessed for eligibility, and 188 were subsequently recruited and randomized.

Eligible patients were skeletally mature adults ( $\geq 18$ years) with an isolated, displaced, closed ankle fracture (Weber classification A, B or C), for whom an operative procedure (rather than conservative treatment) was indicated. Exclusions included patients with open fractures, pilon fractures, previously malunited fractures, peripheral vascular disease precluding tourniquet use, and patients with bilateral fractures or multiple injuries. Patients who were not ambulatory prior to the fracture, unable to complete questionnaires, or with a language barrier that could not be overcome through hospital translation services were also excluded.

Eligible patients were identified on admission and, following plain radiographs to confirm the fracture type, were given a study information sheet and invited to participate in the trial. A research nurse or a research-trained registrar obtained written informed consent, checking that the patient understood what the study involves and providing the opportunity to ask questions. Patients were then allocated to tourniquet or no tourniquet in a 1:1 ratio using an online third-party computerized randomization service at the University of York. Randomization was stratified by age (18-50, over 50), using randomly permuted blocks of 4 and 6.

Fracture type was classified according to the Weber classification [8]. Weber grade A indicates a fracture below the talar dome, grade B denotes a fracture of the distal fibula at the level of the tibiofibular syndesmosis, and grade $\mathrm{C}$ indicates a fracture of the distal fibula proximal to the tibiofibular syndesmosis. Additionally, patients were classified prior to surgery using the American Society of Anesthesiologists (ASA) physical status 
system [9]. Grade I on the classification indicates a normal healthy patient, grade II a patient with mild systemic disease, and grade III a patient with severe systemic disease. The Olerud Molander Ankle Scale (OMAS) score was also recorded [10].

\section{Interventions}

The timing of surgery was determined by the soft tissue swelling. Whenever possible, the operation occurred within 24 hours of injury, but if the tissues were too swollen surgery was delayed until swelling had subsided sufficiently. Operative details were recorded, including duration of operation and tourniquet time. At surgery, the patient was placed supine with a sandbag under the buttock of the affected side. For those patients randomized to have a tourniquet, this was applied above the knee and the leg was prepared, draped and elevated before tourniquet inflation. For all patients, fibular fixation was by plate and screws via a lateral incision, following reduction of the fracture; medial fixation was then undertaken if required. Wounds were closed and a dressing applied. A plaster backslab was applied to all patients to maintain $90^{\circ}$ ankle dorsiflexion and the tourniquet (if used) was deflated. The patient stayed in hospital until assessed as clinically fit for discharge. Patients returned home with walking aids in the backslab. Two weeks post-operation, patients returned to the outpatient clinic to have the wound checked and stitches removed.

Any serious adverse events were recorded and appropriate action taken in accordance with GCP guidelines [11]. Data on hospital LOS, operation duration and intraoperative or immediate post-operative complications were collected or verified from patients' records. Authors HM and DM were among the participating surgeons.

\section{Blinding}

Operating staff were necessarily aware of whether or not a tourniquet was used, but patients were not told until after the study. For determination of LOS, patients were assessed for discharge home by a clinician blinded to tourniquet allocation. Statistical analysis was blind to treatment group allocation.

\section{Outcome measures}

The primary outcome measure, LOS, was defined as the number of days post-operatively that a patient stayed in hospital before being considered clinically fit for discharge, rather than when the patient actually left hospital. Patients fit for discharge on the same day as surgery were ascribed a LOS of zero. Patients were assessed daily and the decision on fitness to go home was taken by at least one clinician not involved in the surgery. Secondary outcomes were the duration of the operative procedure and the occurrence of intraoperative or immediate postoperative adverse events.

\section{Sample size}

The sample size was calculated based on prior data showing a mean LOS (time until fit for discharge) of 4.38 days, with a standard deviation (SD) of 2.72 , for patients having ankle fracture surgery. These data showed LOS to be only moderately positively skewed. A difference in LOS of 1.5 days or greater was considered to be important. In view of the tendency for preliminary data to underestimate the SD in the main trial [12], the observed SD was inflated by $15 \%$ to 3.13 . Accordingly, to detect a 1.5 -day reduction in mean LOS with $90 \%$ power at a 5\% 2-sided significance level, data from a minimum of 93 patients in each comparison group were required (186 in total). 


\section{Statistical analysis}

The primary analysis followed intention-to-treat (ITT) principles and was in accordance with a predetermined statistical analysis plan. The assumptions of statistical models were checked prior to analysis. As the distribution of residuals did not meet the assumptions of linear regression, LOS was compared between groups using Poisson regression, adjusting for Weber classification, ASA grade, sex, BMI, treatment center, and time from fracture to surgery, and including the stratification factor (age) in the model; these covariates were determined a priori. The duration of the operation, for those patients who underwent surgery, was analyzed by analysis of covariance, adjusting for the same covariates. Prior to conducting adjusted analyses, any missing baseline values were imputed, following recent recommendations [13].

An unadjusted ITT analysis and an 'as treated' analysis, whereby patients were analyzed according to the intervention actually received, were carried out as sensitivity analyses [14]. Subgroup estimates of differences in LOS between tourniquet and no tourniquet were calculated for subgroups defined by sex, age (18-50, over 50), Weber classification (B, C) and ASA classification (I, II, III). These subgroup analyses were exploratory and no formal statistical testing was performed.

Statistical significance was set at $p \leq .05$ (2-tailed), and 95\% confidence intervals (CIs) were calculated for between-group estimates. Analysis was conducted in SPSS version 23.

\section{Ethical approval}

Ethical approval for the study was obtained from Staffordshire Research Ethics Committee (Ref 11/WM/0420), after a full review.

\section{Results}

The progression of patients through the trial is shown in a CONSORT diagram (Figure 1). Between August 2012 and August 2015, a total of 471 patients were screened for eligibility, of which 188 were subsequently randomized. Of those randomized to tourniquet, 3 had surgery without a tourniquet (clinical decision $n=2$; randomization missed $n=1$ ) and 3 did not have surgery. Of those randomized to no tourniquet, 19 had surgery with a tourniquet (clinical decision $n=7$; randomization missed $n=10$; other reason $n=2$ ), and one did not have surgery. Following ITT principles, all patients were entered in the analysis.

Patients' baseline characteristics are shown in Table 1. All variables were well balanced across the groups, except that there was a slightly higher percentage of males in the tourniquet group and some imbalance in right versus left fractures.

Figure 2 shows LOS for both treatment groups. Mean length of stay was $1.79 \pm 1.50$ days in the tourniquet group and $1.65 \pm 1.11$ days in the no-tourniquet group; $1.72 \pm 1.32$ days for both groups combined. Median (interquartile range [IQR]) LOS was $1.00(1.00,2.00)$ in both treatment groups. The results of the Poisson regression are shown in Table 3 for the primary analysis (ITT adjusted) and the sensitivity analyses (ITT unadjusted, and astreated). The treatment effect shown is the antilogged coefficient from the regression model and represents a rate ratio, with the no tourniquet group as reference; a ratio greater than 1 therefore indicates a greater LOS with use of a tourniquet. 
In the primary ITT analysis, there was a very small and statistically non-significant difference in LOS; this was on average $7.2 \%$ greater in the tourniquet group. The difference in LOS was much smaller than the 1.5-day difference considered to be clinically important. The sensitivity analysis based on an unadjusted ITT analysis gave a similar non-significant result: LOS was estimated as $8.4 \%$ greater in the tourniquet group. In the as-treated sensitivity analysis, the difference was again small and non-significant, but favored the tourniquet group: LOS was on average 3.8\% shorter than in the no-tourniquet group.

Figure 3 shows mean differences in LOS from the adjusted ITT analysis by subgroups defined by sex, age (18-50, over 50), Weber classification (B, C) and ASA grade $(1,2,3)$. The estimates for the Weber classification subgroups were very similar to the overall estimate. Estimates for male patients and those over 50 appeared to favor non-use of tourniquet, while those for female patients and those aged 18-50 appeared to favor tourniquet use. The subgroups defined by ASA grade show some variation, but it should be noted that there are only 5 patients in ASA grade 3 and the estimate for this subgroup is therefore very imprecise (as indicated by the very wide $\mathrm{CI}$ ).

The mean duration of the operative procedure was $71.98 \pm 25.54$ minutes for the tourniquet group and $76.37 \pm 27.71$ minutes for the no-tourniquet group. The covariateadjusted mean difference in the ITT analysis (no tourniquet minus tourniquet) was 3.03 minutes $(95 \% \mathrm{CI}-4.96,11.02 ; p=.455)$. The unadjusted ITT mean difference was 4.39 minutes $(95 \% \mathrm{CI}-3.35,12.12 ; p=.264)$. In the as-treated sensitivity analysis, the mean duration of the operative procedure was $73.11 \pm 23.64$ minutes for the tourniquet group $(\mathrm{n}=107)$ and $76.58 \pm 29.51$ minutes for the no-tourniquet group $(\mathrm{n}=77)$. The covariateadjusted mean difference was very similar to that of the ITT analysis at 2.35 minutes (95\% CI -5.62, 10.31; $p=.562)$.

Owing to the small effect of tourniquet use on LOS, an additional unplanned analysis was undertaken to determine the relative effect of other potential predictors. The following variables, in addition to tourniquet use, were entered in a Poisson regression model, following a check for collinearity: age, BMI, time from fracture to surgery, time to operation, duration of operation, OMAS score, sex, Weber classification, ASA grade, regular smoker, mechanism of fracture (see Table 1 for categorical variable values). As these represent a mixture of dichotomous, multinomial and numeric variables, rate ratios from the regression analysis are not comparable. The $P$ value from a likelihood ratio test of each variable was therefore used as an indirect index of its strength as a predictor of LOS. Table 3 gives the results of the analysis. Variables are presented in ascending order of their associated $P$ values, and hence in descending order of predictive strength. ASA grade (higher grade predicts longer LOS) and time from fracture to surgery (shorter time predicts longer LOS) were significant independent predictors. Ignoring statistical significance, BMI, age and duration of operation were additionally stronger predictors than tourniquet use. It should be noted that no sample size calculation had been performed for these unplanned analyses.

\section{Adverse events}

Seven patients experienced intraoperative complications (Table 4); $2(2.2 \%)$ in the tourniquet group and $5(5.4 \%)$ in the no-tourniquet group (odds ratio with no tourniquet as reference category 0.396 (95\% CI 0.075, 2.093); $p=.444$, Fisher's exact test). One patient experienced severe pain postoperatively, when a cast was applied (an alternative cast was subsequently applied); this was considered to be unrelated to the study 
interventions. No patients required admission to an intensive care unit, and none were readmitted for further surgery on the affected ankle. There was one serious adverse event. A patient in the no-tourniquet group required prolonged hospitalization following desaturation in the recovery room; this was considered to be unrelated to the study interventions.

\section{Discussion}

The non-use of a tourniquet did not produce a statistically significant or clinically important difference in the primary outcome measure, and this study did not therefore confirm previous evidence that use of a tourniquet increases length of stay [5-7]. In the exploratory analysis, the only 2 variables that significantly affected LOS were the ASA grade, which is a measure of pre-existing health before the ankle injury has occurred, and time from fracture to surgery. Those patients with an ASA grade of 1 had a mean hospital LOS of 1.35 days and those with an ASA 2 had a mean hospital LOS of 2.24 days. $60 \%$ of patients were ASA grade 1 and $35.5 \%$ of patients were ASA grade 2 . Those patients with a shorter time from fracture to surgery had a longer LOS. The reason for this is unclear from our data.

The mean overall length of stay in our study of 1.72 days was considerably shorter than the mean of 4.38 days in the preliminary data that we had collected, and shorter than the mean 3.50 and 4.50 days of postoperative stay reported, respectively, by Lloyd et al [15] and Singh et al [16]. Consequently, the targeted reduction in length of stay of 1.5 days appeared to be unrealistic. It is not clear why length of stay was so much shorter in our study. It does not appear to reflect the decision to assess length of stay in terms of medical fitness for discharge, as the mean length of stay until actual discharge was similar, at 1.99 days. Instead, it may reflect changes in practice within the participating hospitals since the preliminary data were collected.

It has recently been reported that patients who have surgery for closed ankle fractures within 24 hours of injury have shorter length of stay than those operated on later [16]. Our data do not confirm this. The mean length of stay for those operated on within 24 hours (1.89 days; $n=46$ ) was greater than that for those operated on later (1.66 days; $n$ =142).

There was a higher rate of protocol deviation in the no-tourniquet group (a tourniquet was used for 19 patients) than in the tourniquet group (only 3 patients had no tourniquet). This may reflect the surgeon's personal preference for use of a tourniquet to provide a bloodless field. The as-treated analysis, which reflects this protocol deviation, showed LOS to be slightly shorter with tourniquet use, but the difference is nonsignificant. Confirming previous findings [5], tourniquet use did not have a significant effect on operative time, although duration of operation was slightly shorter in the nontourniquet group than in the tourniquet group (72.34 vs 76.37 minutes).

The strengths of our study include the fact that we have a complete set of data on length of stay and on operation time for patients undergoing surgery, and statistical analysis was blinded, allowing us to reach unbiased conclusions regarding LOS. All staff assessing patients' fitness for discharge were blinded to tourniquet use, reducing the risk of bias in clinical decision making. Broad inclusion criteria assist the generalizability of our findings. The availability of other medical data, including ASA grade, time from injury to surgery has allowed us to perform further exploratory statistical analysis on other factors that affect length of stay, though as these were unplanned analyses for which the study 
was not specifically powered, they must be interpreted with caution. Similarly, the subgroup analyses were exploratory and should be interpreted accordingly. The high rate of protocol deviation in the no-tourniquet group suggests, in some instances, a lack of clinical equipoise, though the as-treated analysis indicates this is unlikely to have seriously biased the statistical comparisons.

In conclusion, our study has demonstrated that hospital LOS following operative management of closed ankle fractures in skeletally mature adults is not significantly affected by the use of a tourniquet during surgery. Pre-injury medical condition of the patient appeared to be the single most influential factor identified affecting hospital length of stay.

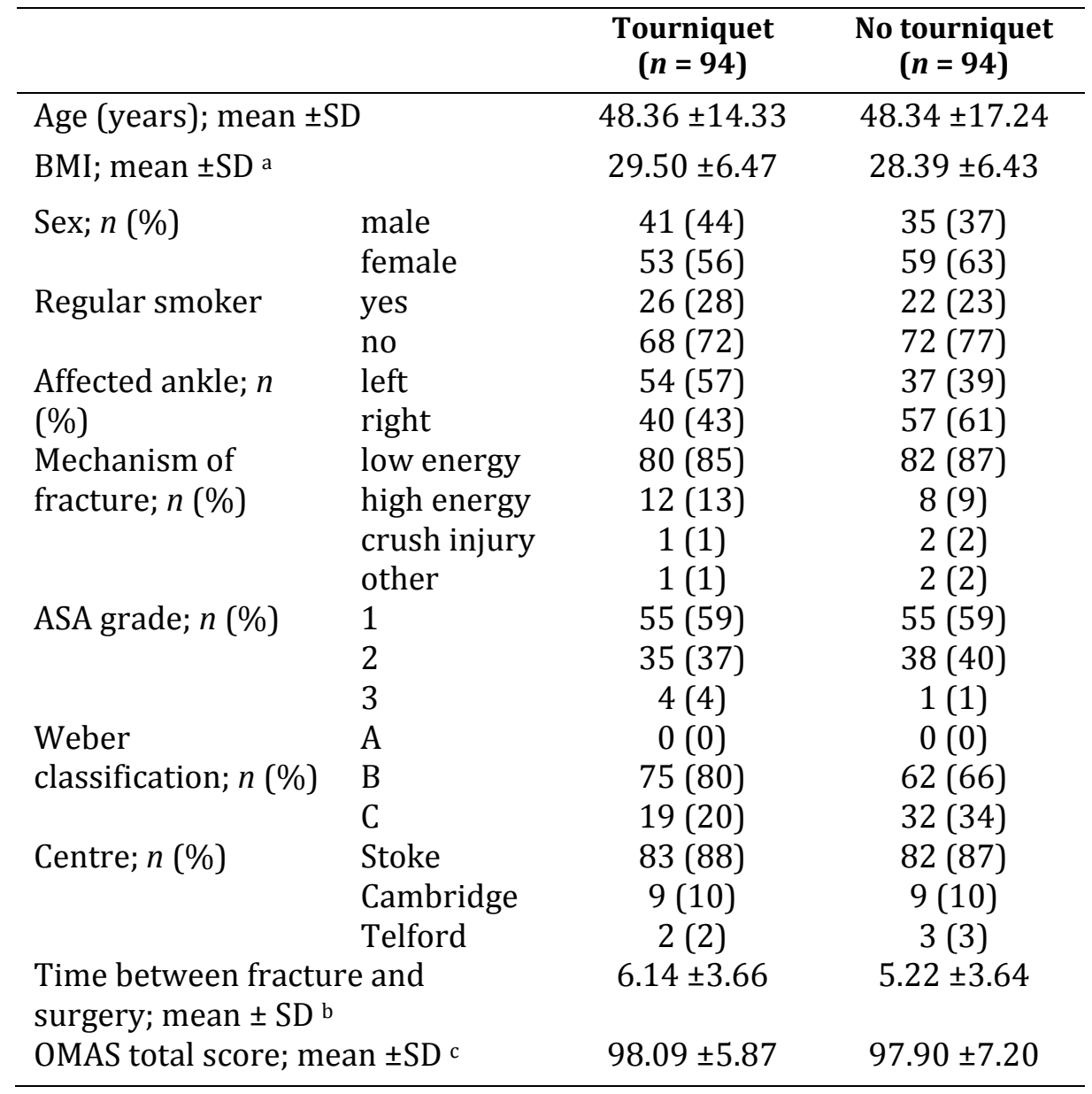

a 19 missing values, $n_{1}=86, n_{2}=83$; b 3 patients did not receive surgery, so time to operation calculated in terms of when surgery was scheduled; 1 missing value; $n_{1}=94, n_{2}=93$; OMAS = Olerud Molander Ankle Scale.

Table 1. Baseline characteristics $(N=186)$. 


\begin{tabular}{|c|c|c|c|c|c|}
\hline Analysis & $\begin{array}{c}\text { Tourniquet } \\
\text { (mean; days) }\end{array}$ & $\begin{array}{c}\text { No } \\
\text { tourniquet } \\
\text { (mean; days) }\end{array}$ & $\begin{array}{l}\text { Effect } \\
\text { (rate } \\
\text { ratio) }{ }^{a}\end{array}$ & $\begin{array}{l}95 \% \text { confidence } \\
\text { interval }\end{array}$ & $\begin{array}{c}p \\
\text { value }\end{array}$ \\
\hline \multicolumn{6}{|l|}{ Primary: } \\
\hline $\begin{array}{l}\text { ITT analysis, } \\
\text { adjusted } \mathrm{b}\end{array}$ & 1.74 & 1.62 & 1.072 & $0.854,1.346$ & .549 \\
\hline \multicolumn{6}{|l|}{ Sensitivity: } \\
\hline $\begin{array}{l}\text { ITT analysis, } \\
\text { unadjusted }\end{array}$ & 1.79 & 1.65 & 1.084 & $0.871,1.348$ & .470 \\
\hline $\begin{array}{l}\text { As-treated analysis, } \\
\text { adjusted b,c }\end{array}$ & 1.65 & 1.71 & 0.962 & $0.764,1.212$ & .744 \\
\hline
\end{tabular}

a Reference group is no tourniquet; b Adjusted for Weber classification, sex, BMI, treatment center, time from fracture to surgery, sex, and age; ${ }^{c}$ based on $n=107$ and $n=77$ for tourniquet and no-tourniquet groups respectively.

Table 2. Results of the Poisson regression analysis on length of stay $(N=186)$.

\begin{tabular}{lccc}
\hline Predictor & $\begin{array}{c}\text { Likelihood } \\
\text { ratio chi- } \\
\text { square }\end{array}$ & $\begin{array}{c}\text { Degrees of } \\
\text { freedom }\end{array}$ & $\boldsymbol{p}$ value \\
\hline ASA grade & 10.571 & 2 & .005 \\
Time from fracture to surgery & 4.996 & 1 & .025 \\
BMI & 3.024 & 1 & .082 \\
Age & .879 & 1 & .349 \\
Duration of operation & .826 & 1 & .363 \\
Tourniquet use & .806 & 1 & .369 \\
Sex & .507 & 1 & .476 \\
Weber classification & .068 & 1 & .795 \\
Mechanism of fracture & .826 & 3 & .843 \\
Regular smoker & .015 & 1 & .904 \\
OMAS score & $<.001$ & 1 & .999 \\
\hline
\end{tabular}

Table 3. Predictors of length of stay $(N=169)$.

\begin{tabular}{lcc}
\hline Complication & $\begin{array}{c}\text { Tourniquet } \\
\text { group }(\boldsymbol{n}=\mathbf{9 1})\end{array}$ & $\begin{array}{c}\text { No-tourniquet } \\
\text { group }(\boldsymbol{n}=\mathbf{9 3})\end{array}$ \\
\hline Vascular injury & 0 & 1 \\
Blood loss $(250 \mathrm{ml})$ & 0 & 1 \\
Aspiration & 0 & 1 \\
Drill bit snapped & 1 & 1 \\
Other equipment/staff issues & 1 & 1 \\
\hline
\end{tabular}

Table 4. Intraoperative complications in patients undergoing surgery $(N=184)$. 


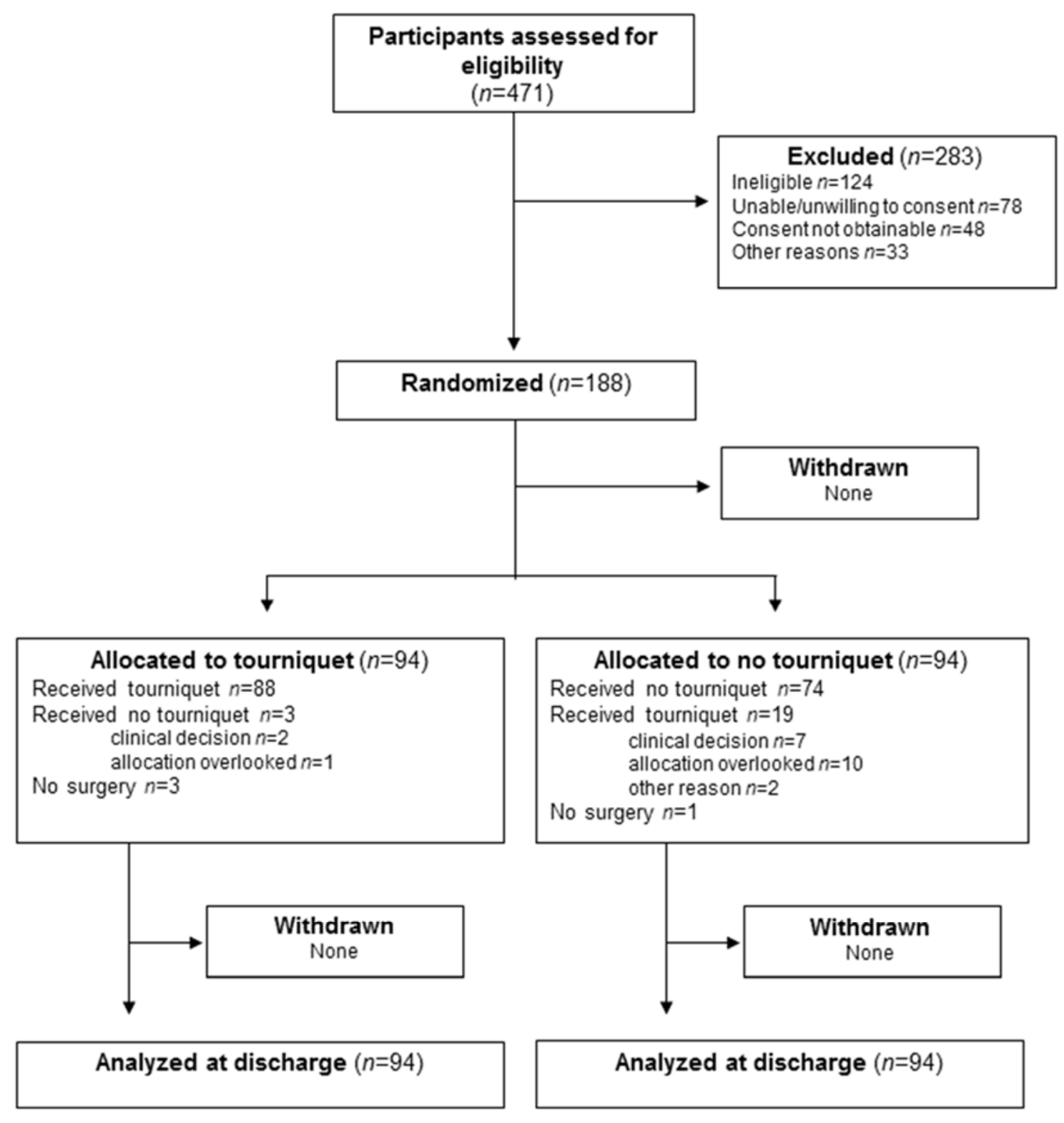

Figure 1. CONSORT diagram showing the progression of patients through the trial. 


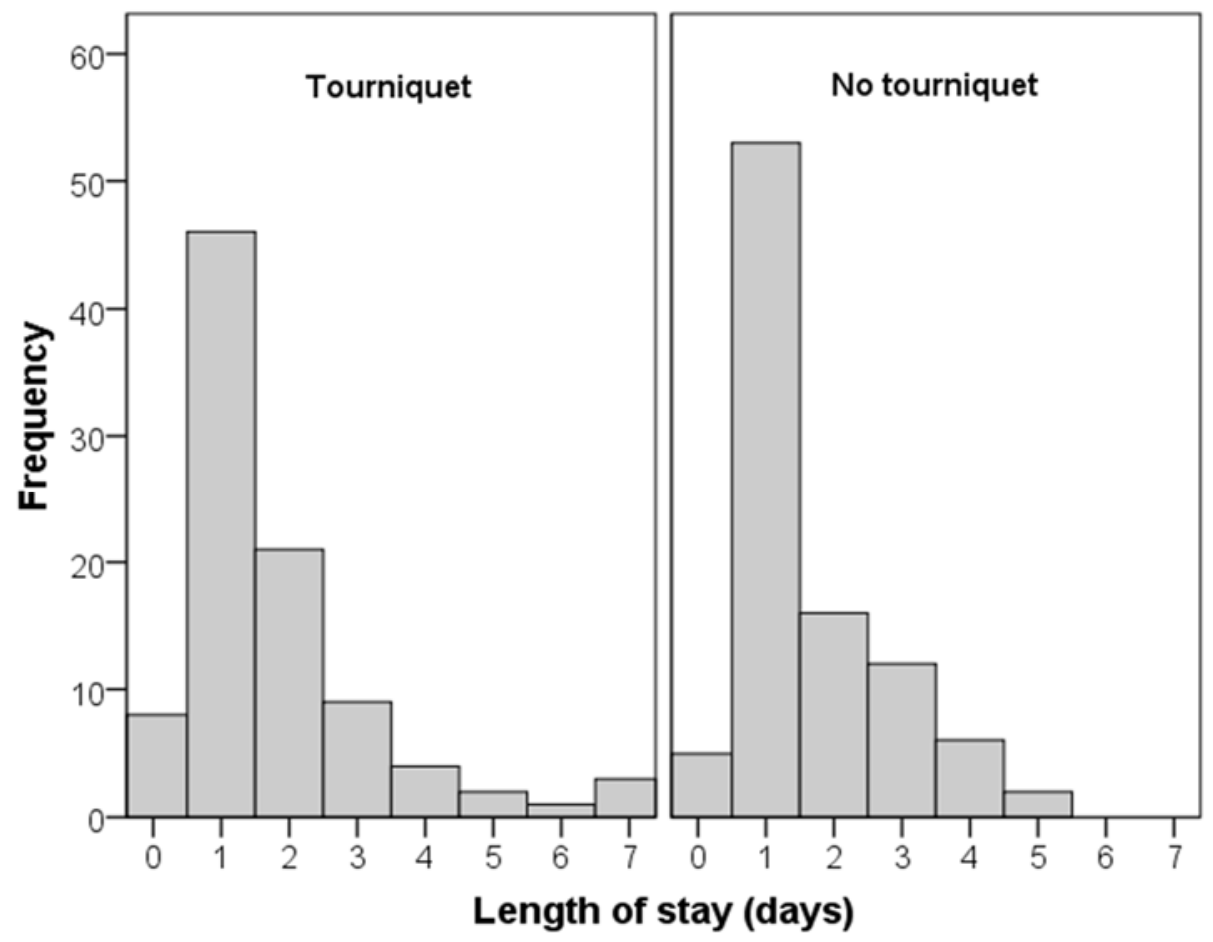

Figure 2. Length of stay in the tourniquet and no-tourniquet groups.

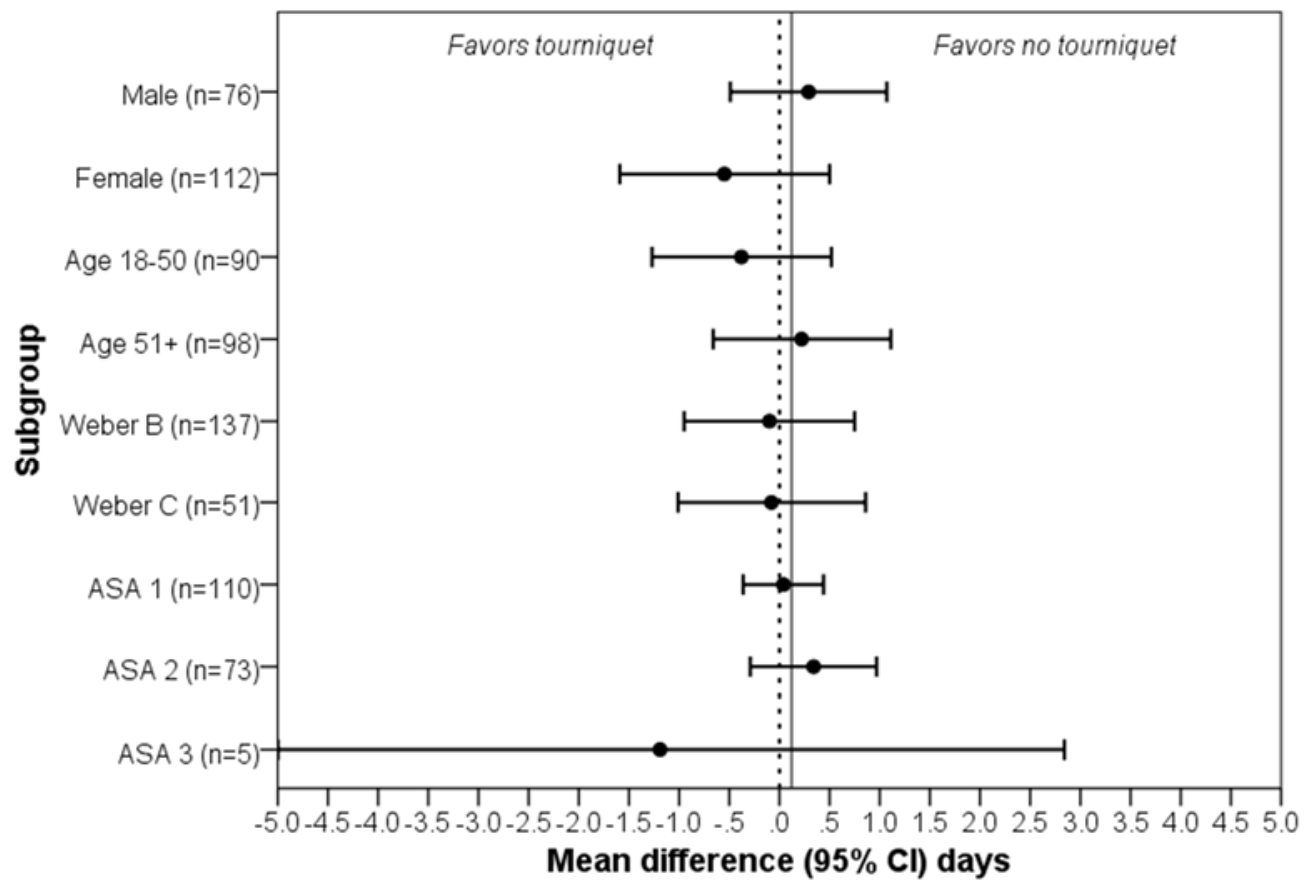

Figure 3. Length of stay for subgroups. Figures are mean difference in days (tourniquet group minus no-tourniquet group) with $95 \%$ confidence intervals. Negative differences favor the tourniquet group; positive differences favor the no-tourniquet group. The solid vertical reference line indicates the overall mean difference. The dashed vertical reference line indicates a mean difference of zero. 


\section{Funding}

This paper represents independent research funded by the National Institute for Health Research (NIHR) under its Research for Patient Benefit Programme (project number PBPG-0610-22381). The views expressed are those of the authors and do not necessarily reflect those of the NHS, NIHR, or the Department of Health and Social Care. It was sponsored by University Hospitals of North Midlands NHS Trust.

\section{Acknowledgments}

We would like to acknowledge assistance from Dr Heather Smith for input to the design of the study and assistance in securing funding, Gareth Hurley for input to the design of the study, Dr Sarah Griffiths for her help in the study set-up, and Dr Naheed Akhtar for her assistance in the management of the study. We also thank the participating surgeons.

\section{Declarations of interest: none}

Financial disclosure: none

\section{References}

1. Daly PJ, Fitzgerald RH Jr, Melton LJ, Ilstrup DM. Epidemiology of ankle fractures in Rochester, Minnesota. Acta Orthop Scand 58(5):539-544, 1987.

2. Younger ASE, Kalla TP, McEwen JA. Sourvey of tourniquet use in orthopaedic foot and ankle surgery, Foot Ankle Int 26(3):208-217, 2005.

3. Kam PC, Kavanagh R, Yoong. The arterial tourniquet: pathophysiological consequences and anaesthetic implications. Anaesthesia 56(6):534-545, 2001.

4. Lee MS, Grossman JP. Complications in Foot and Ankle Surgery: Management Strategies. New York: Springer; 2017.

5. Smith TO, Hing CB. The efficacy of the tourniquet in foot and ankle surgery? A systematic review and meta-analysis. Foot Ankle Surg. 16(1):3-8, 2010.

6. Konrad G, Markmiller M, Lenich A, Mayr E, Rüter A. Tourniquets may increase postoperative swelling and pain after internal fixation of ankle fractures. Clin Orthop Relat Res 433:189-194, 2005.

7. Maffulli N, Testa V, Capasso G. Use of a tourniquet in the internal fixation of fractures of the distal part of the fibula. A prospective, randomized trial. J Bone Joint Surg Am 75(5):700-703, 1993.

8. Weber BG, Colton C. Malleolar fractures. In: Müller ME, Allgöwer M, Schneider R, Willenegger H, eds. Manual of Internal Fixation: Techniques Recommended by the AO-ASIF Group. Third ed. Berlin: Springer-Verlag; 1991: 595-612.

9. American Society of Anesthesiologists. ASA Physical Status Classification System. Oct 2014; Available from: https://www.asahq.org/resources/clinical- 
information/asa-physical-status-classification-system (date accessed 12 November 2017)

10. Olerud C, Molander H. A scoring scale for symptom evaluation after ankle fracture. Arch Orthop Trauma Surg 103(3):190-194, 1984.

11. ICH. International Council for Harmonisation of Technical Requirements for Pharmaceuticals for Human Use. Integrated addendum to ICH E6 (R1); Guideline for good clinical practice ICH Nov 2016; Available from:

https://www.ich.org/fileadmin/Public Web Site/ICH Products/Guidelines/Effic acy/E6/E6 R2 Step 4.pdf (date last accessed 12 November 2017).

12. Vickers AJ. Underpowering in randomized trials reporting a sample size calculation. J Clin Epidemio. 56(8):717-720, 2003.

13. White IR, Thompson SG. Adjusting for partially missing baseline measurements in randomized trials. Stat Med 2005;24(7):993-1007, 2005.

14. Wright CC, Sim J. Intention-to-treat approach to data from randomized controlled trials: a sensitivity analysis. J Clin Epidemiol 56(9):833-842, 2003.

15. Lloyd JM, Martin R, Rajagopolan S, Zieneh N, Hartley R. An innovative and costeffective way of managing ankle fractures prior to surgery - home therapy. Ann $R$ Coll Surg Engl 92(7):615-618, 2010.

16. Singh RA, Trickett R, Hodgson P. Early versus late surgery for closed ankle fractures. J Orthop Surg (Hong Kong) 23(3):341-344, 2015. 